
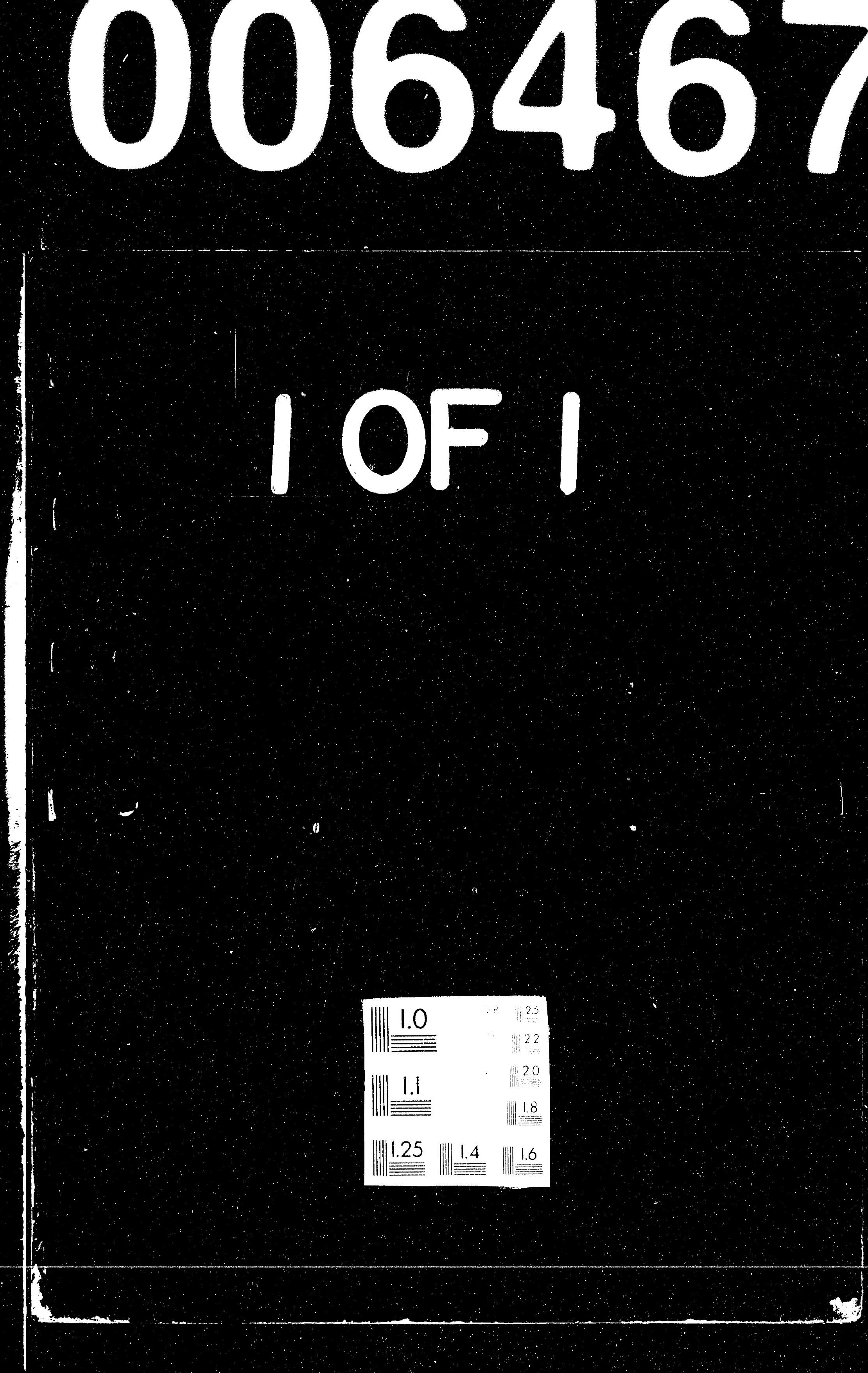
Annual Report On

Mechanisms and Enhancement of Flame Stabilization

(Grant DE-FG02-89ER13988)

For the Period of January 15, 1992 to January 14, 1993

Submitted To:

Department of Energy

Division of Engineering, Mathematics and Geosciences

Office of Basic Energy Sciences

Submitted By:

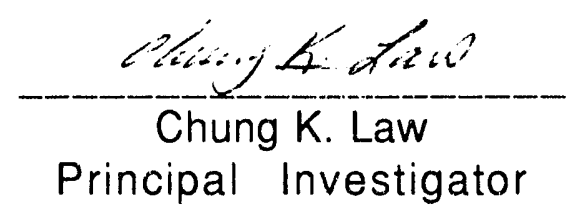

Princeton University

Department of Mechanical and Aerospace Engineering

Princeton, New Jersey 08544 


\section{Summary of Research Accomplishments}

During the reporting period, useful contributions have been made in understanding the structure of laminar premixed and diffusion flames, with emphasis on the influence of aerodynamics and chemical kinetics. These contributions inclide (1) derivation of the missing closure condition for the activation energy asymptotic analysis of premixed flames, (2) identification of a dual extinction mode for radiation-affected flames, (3) formulation of a unified theory of fundamental flammability limits, and (4) demcinstration that flame stabilization can be achieved in the absence of heat loss. These investigations have been conducted via experimental, analytical and computational approaches, with strong coupling between the individual components.

The highlights of these accomptishments will be briefly discussed in the following. Details of these work can be found in the various journal-class papers listed in the next section.

\section{Closure in Activation Energy Asymptotics of Premixed Flames}

Although the method of activation energy asymptotics has been extensively employed in flame structure studies, a unified and rigorous closure of the methodology when applied to premixed flame analysis does not appear to have been identified. While various closure schemes have been explicitly or implicitly invoked, they were applied with no or minimum mathematical and/or physical justification.

In the present study we first argued that since the reaction rate function was not spatially uniform, it was not correct to approximate it as a delta function in the reaction-sheet limit, as was invariably done. Neglecting the higher-order singularities through imposition of arbitrary closure schemes could therefore lead to $O(1)$ errors in the solution. It was subsequently demonstrated that closure, to the needed $O(\varepsilon)$ accuracy in the solution, could be achieved by locating the reaction sheet at the "center of reaction", which was based on the first moment of the reaction rate function about the location of its maximum point. A generalized analysis of the reaction region then showed that this closure relation was equivalent to the requirement that the outer solutions of temperature and concentration be continuous to $O(\varepsilon)$ at the reaction sheet. The importance of closure was further demonstrated by applying the present and 
various literature closure expressions for a comprehensive analysis of the stabilization and extinction of a nonequidiffusing counterflow flame, with the stagnation surface being either adiabatic or nonadiabatic. It was shown that quantitatively and qualitatively different solutions could result depending on the applied closure.

This work is reported in Publication No. 1.

\section{Dual Extinction States of Radiation-Affected Flames}

Existing understanding of flame extinction is based on the concept of the Damkohler number, $\mathrm{Da}$, defined as the ratio of the characteristic flow time to a characteristic reaction time in the flame. Thus for a steadily-burning flame, decreasing $\mathrm{Da}$ reduces the available time for reaction and thereby promotes extinction. The viability of this concept has been extensively demonstrated both theoretically and experimentally. Such an understanding, however, is based on a conservative system in that in the reaction-sheet limit the flame temperature is the adiabatic flame temperature. A combustion, system is nevertheless inherently nonadiabatic due to radiative loss from the flame as well as the hot surfaces which may be present in the system.

The subtlety here is that radiative heat loss is a volumetric process and its loss rate frequently increases with increasing system dimension and thereby the flow time. Thus while increasing the system Da allows more time for chemical reaction to proceed, it also leads to more heat loss and therefore reduces the flame temperature. With sufficient reduction in the flame temperature, extinction can conceivably also occur even with the increase in the time available for reaction.

Combining the above considerations, it is therefore reasonable to expect that steady burning can exist only for a range in $\mathrm{Da}$, bounded by a kineticallycontrclled $\mathrm{Da}$ on the lower limit and a loss-controlled $\mathrm{Da}$ on the lipper limit. When these two limits coincide, steady burning is not possible.

The above concept was verified from a theoretical study in which heat loss from a gasifying surface was considered. This study is reported in Publication No 2.

\section{Theory of Fundamental Flammability Limits}

While "flammability limit" has a long and prominent history in the description of combustion phenomena, a clear and unique fundamental 
definition, which will also allow for its unambiguous theoretical and experimental determination, has yet to be identified. As a consequence, the term "flammability limit" has been widely and loosely applied to diverse situations of unsustainable combustion, many of which represent only limits of flame extinction. Clearly, if flammability limit is indeed a useful fundamental concept, then a combustible fuel/oxidizer system can have only a lean limit and a rich limit, which occur at two distinct concentrations. As such, these two flammabilty limits should be unique physico-chemical properties of a combustible system, independent of such external influences as conductive and convective heat loss, aerodynamic straining, gravity-related phenomena, etc.

In view of the above considerations, the configuration based on which flammability limit can be usefully defined is the state at which steady propagation of the one-dimensional, planar premixed flame in the doublyinfinite domain fails to be possible. The two omnipresent, system-independent processes which could cause extinction of such a flame are radiative loss and the chain branching and termination reactions in the chemical reaction mechanism. While radiative loss is an obvious extinction mechanism, extinction due to chain reactions can be anticipated from the following consideration. That is, as the flammability limit is approached, the continuous reduction of the flame temperature weakens the temperature-sensitive branching reaction relative to the termination reaction which is in general less temperature sensitive. This causes a slowdown in the overall reaction rate. Eventually the flame can be so weakened that it is extinguished by system perturbations which are invariably present.

In this study the flammability limits of many mixtures were first experimentally determined by measuring the extinction limits of stretched, counterflow flames and extrapolating the results to zero stretch. Extensive determinations were conducted for the lean and rich limits for mixtures of methane, ethane, ethylene, acetylene, and propane with air, and for the effects of dilution, inert substitution, and chemical additives. In the theoretical investigation, the one-dimensional planar flame propagation was first simulated with detailed chemistry and transport, but without radiative heat loss. The sensitivity of the dominant chain termination reaction to the dominant chain branching reaction was continuously monitored as the concentration of the deficient reactant was reduced. Extinction was assumed to occur when the normalized sensitivity reached unity. The predicted values 
agreed well with the experimental data. Furthermore, a study of the characteristics of the chain mechanisms for different mixtures also explained some well-known anomalies concerning flammability limits. This work was reported last year.

In the current report period, radiative heat loss was included, and the characteristic extinction turning point was obtained. A particularly significant result from this study was that, at the state of the heat-loss induced turning point, the normalized sensitivity of the chain reactions also assumed the unity value. This therefore provides a unified interpretation of the flammability limits based on both the physical process of heat loss and the chemical process of chain termination. That is, as the flammability limit is approached, the overall reaction is weakened due to the increased importance of the chain termination reaction. The heat release rate is eventually reduced to such an extent that radiative heat loss becomes important and leads to flame extinction. This work is reported in Publication No. 3.

\section{Flame Stabilization and Blowoff}

We have made two contributions in this endeavor. First, we have provided a comprehensive review on the mechanisms of flame stabilization in subsonic and supersonic flows. The review classified and discussed current understanding on the fundamental physico-chemical mechanisms governing the structure and stabilization mechanisms of premixed and diffusion flames in subsonic and supersonic laminar and turbulent flows. Specific topics discussed included the ignition of combustibles in homogeneous and diffusive media, the extinction of premixed and diffusion flames through reactant leakage, heat loss, and aerodynamic stretching, the stabilization and liftoff of burner-stabilized and rim-stabilized flames, and the various proposed mechanisms for the stabilization and blowout of jet diffusion flames. The fundamental similarities and differences between the various critical phenomena are indicated, and potential research topics suggested. This review is listed as Publicaticn No. 4.

The second project basically started our research endeavor on the understanding of flame stabilization and blowoff. To appreciate the approach we have undertaken, we first note that the mechanism with which a Bunsen flame is stabilized at the burner rim is generally considered to be well established. The concept of stabilization is based on the existence of a dynamic balance between the local flow velocity and flame velocity at a certain point on 
the- flame surface, and the ability of the flame to adjust its velocity, and thereby the location of stabilization, through heat loss to the rim. Blowoff occurs when such a balance cannot be achieved everywhere over the flame surface. This mechanism has served as the fundamental concept in flame stabilization in other situations.

In the stabilization mechanism just described, heat loss to the burner rim is the only factor that can modify the flame speed. However, recent studies on the general structure and response of laminar flames have conclusively demonstrated that the burning intensity of the flame can also be significantly modified by the extent of flow nonuniformity, flame wrinkling and unequal molecular diffusivity of the system. The presence of these additional factors offers enhanced flexibility for the flame to achieve stabilization, and significantly enriches the phenomena of flame stabilization and blowoff.

Perhaps the most intriguing question to ask then is whether flame stabilization can be achieved in the absence of heat loss, through the modification of the flame burning intensity by other factors. To explore this possibility, an inverted flame experiment was conducted. Here a thin rod was placed coaxially in a uniform flow of combustible mixture (see Fig. 8). If the flow velocity was not large, upon ignition an inverted (Bunsen) flame could be established downstream of the trailing edge of the rod. With continuous increase in the flow velocity, the flame would recede from the rod and would be eventually blown off. The extent of heat loss from the flame base to the stabilizing rod was determined by measuring the temperature in the stabilizing region between the flame base and the rod by using both thermocouples and laser Raman spectroscopy.

The results showed that when the flame base is moderately away from the rod, the amount of heat transfer to the rod was essentially negligible. This therefore demonstrated that flame stabilization could be accomplished through flame stretch and nonequidiffusion effects, in the absence of heat loss. A theory was subsequently formulated based on the dynamic balance between the local flame and flow velocities. The results showed that such a balance could indeed be accomplished.

This work is reported in Publication No. 5. 


\section{Publications}

1. "On closure in activation energy asymptotics of premixed flames," by C.K. Law, B.H. Chao and A. Umemura, Combustion Science and Technology 88,pp. 59-88 (1992).

2. "Asymptotic theory of flame extinction with surface radiation," by B.H. Chao and C.K. Law, Combustion and Flame 92, pp. 1-24 (1993).

3. "A unified chain-thermal theory of fundamental flammability limits," by C.K. Law and F.N. Egolfopoulos, Twenty-Fourth Symposium (International) on Combustion, The Combustion Institute, Pittsburg, PA, pp. 137-144 (1992).

4. "Mechanisms of flame stabilization in subsonic and supersonic flows," by C.K. Law, Major Topics in Combustion (Eds.: M.Y. Hussaini, A. Kumar and R.G. Voigt), Springer-Verlag, pp. 201-236 (1992).

5. "On adiabatic stabilization of inverted flames," by C.J. Sung, C.K. Law and A. Umemura, Twenty-Fourth Symposium (International) on Combustion, The Combustion Institute, Pittsburg, PA, pp. 205-212 (1992). 

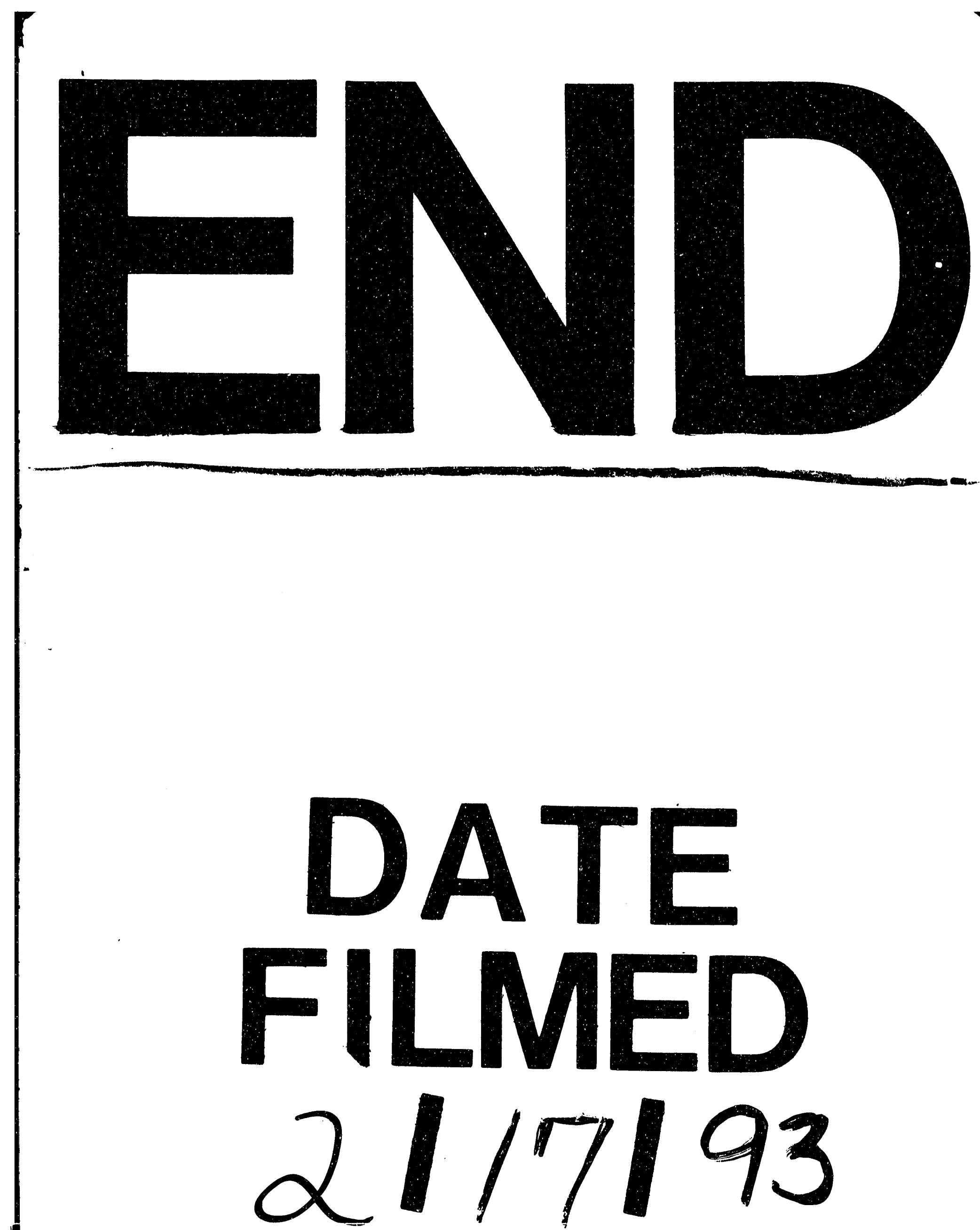
